lustitia Socialis. Revista Arbitrada de Ciencias Jurídicas.

Año V. Vol. V. №3. Edición Especial. 2020-III:

Universidad Regional Autónoma de los Andes

Hecho el depósito de Ley: FA2016000064

ISSN: 2542-3371

FUNDACIÓN KOINONIA (F.K). Santa Ana de Coro, Venezuela

Iván Xavier León-Rodríguez; Pamela Nicohol Villarreal-Vivanco

http://dx.doi.org/10.35381/racji.v5i3.1140

\title{
Carga procesal en audiencias en materia de niñez y adolescencia
}

\section{Procedural burden in hearings on children and adolescents}

\author{
Iván Xavier León-Rodríguez \\ us.ivanleon@uniandes.edu.ec \\ Universidad Regional Autónoma de los Andes, Santo Domingo \\ Ecuador \\ https://orcid.org/0000-0002-3725-988X \\ Pamela Nicohol Villarreal-Vivanco \\ ds.pamelanvv@uniandes.edu.ec \\ Universidad Regional Autónoma de los Andes, Santo Domingo \\ Ecuador
}

Recibido: 31 de octubre de 2020

Revisado: 10 de octubre de 2020

Aprobado: 05 de diciembre de 2020

Publicado: 10 de diciembre de 2020 
lustitia Socialis. Revista Arbitrada de Ciencias Jurídicas.

Año V. Vol. V. N³. Edición Especial. 2020-III:

Universidad Regional Autónoma de los Andes

Hecho el depósito de Ley: FA2016000064

ISSN: 2542-3371

FUNDACIÓN KOINONIA (F.K). Santa Ana de Coro, Venezuela

Iván Xavier León-Rodríguez; Pamela Nicohol Villarreal-Vivanco

\title{
RESUMEN
}

La investigación artículo tuvo por objetivo analizar la incidencia en el despacho oportuno de audiencias en materia de Niñez y Adolescencia en contraposición de la carga procesal que tienen los Magistrados del Cantón de Santo Domingo - Ecuador. Se desarrolló metodológicamente desde una perspectiva descriptiva documental con diseño bibliográfico, lo cual permitió estudiar una población de 6916 causas ingresadas en el año 2017, en el año 2018 de 5818 y en 2019 fue de 5895 en materia de niñez y adolescencia. Se ha evidenciado el incumplimiento de los principios de celeridad, eficacia y eficiencia en la administración de justicia de niñez, esto debido a la excesiva carga procesal asignada a los jueces, que en nuestra provincia la referida unida judicial apenas cuenta con nueve jueces, para atender 4.869 causas anuales.

Descriptores: Mediación; administración de justicia; procedimiento legal. (Palabras tomadas del Tesauro UNESCO).

\begin{abstract}
The objective of the article investigation was to analyze the incidence in the timely dispatch of hearings on Childhood and Adolescence as opposed to the procedural burden that the Magistrates of the Canton of Santo Domingo - Ecuador have. It was methodologically developed from a descriptive documentary perspective with bibliographic design, which allowed the study of a population of 6,916 cases admitted in $2017,5,818$ in 2018, and in 2019 it was 5,895 in childhood and adolescence. Failure to comply with the principles of speed, effectiveness and efficiency in the administration of childhood justice has been evidenced, due to the excessive procedural burden assigned to judges, which in our province the aforementioned judicial unit barely has nine judges, to attend 4,869 annual causes.
\end{abstract}

Descriptors: Mediation; administration of justice; legal procedure. (Words taken from the UNESCO Thesaurus). 
lustitia Socialis. Revista Arbitrada de Ciencias Jurídicas.

Año V. Vol. V. №3. Edición Especial. 2020-III:

Universidad Regional Autónoma de los Andes

Hecho el depósito de Ley: FA2016000064

ISSN: 2542-3371

FUNDACIÓN KOINONIA (F.K). Santa Ana de Coro, Venezuela

Iván Xavier León-Rodríguez; Pamela Nicohol Villarreal-Vivanco

\section{INTRODUCCIÓN}

La relación del concepto de carga con el impulso procesal radica en que el juicio avanza también mediante cargas impuestas a las partes. Principio Procesal. Según el Diccionario de la Real Academia Española de la Lengua (2016, pág. 95) el término Principio significa; punto que se considera como primero en una extensión o cosa, base, origen o razón fundamental sobre la cual se procede discurriendo en cualquier materia. En la doctrina nacional enseña que los principios procesales: Son aquellas reglas de valoración que se deducen del ordenamiento jurídico y que sirven de fundamento para la interpretación y aplicación de las normas procesales en atención a un criterio axiológico primaria: la realización de Justicia. (Hernández-Villareal \& Ortiz, 2010).

De ese modo se abre paso al principio de celeridad, este principio influye en una administración de justicia penal rápida para resolver la conflictividad existente entre los derechos del ofendido y del procesado y la correcta recolección de los medios de prueba para evitar que se afectan los derechos de las personas y que estos medios de prueba no desaparezcan o se contaminen. (Puppio, 2012, p. 25)

El proceso debe ser rápido y sin dilaciones injustificadas si se considera la trascendencia de todo lo que comprometen las partes en él: la libertad, sus bienes, la expectativa de una condena, sus familias, su futuro, su vida misma. El proceso debe ser oportuno para la sociedad que exige ver como se investiga y condena el hecho que la conmovió; igualmente deberá ser célere por consideración al procesado que observa cómo se va dilatando el tiempo sin que se resuelva su situación (Gutiérrez-Campoverde, et al., 2019). Es la manifestación concreta del principio de economía procesal por razón de tiempo. Mediante este principio se busca que el proceso no se dilate más tiempo del necesario, vale decir, el proceso debe desarrollarse en los plazos establecidos por Ley, ni rápido ni lento, sino respetando el debido proceso. La celeridad judicial es una de las discusiones a que más alusión se hace en los últimos tiempos. Pareciera que la sociedad toda se haya volcado a plantear esta temática y no solamente el estamento de los juristas. Esto revela que la sociedad está ávida y ansiosa de soluciones y de planteamientos útiles en 
lustitia Socialis. Revista Arbitrada de Ciencias Jurídicas.

Año V. Vol. V. N³. Edición Especial. 2020-III:

Universidad Regional Autónoma de los Andes

Hecho el depósito de Ley: FA2016000064

ISSN: 2542-3371

FUNDACIÓN KOINONIA (F.K). Santa Ana de Coro, Venezuela

Iván Xavier León-Rodríguez; Pamela Nicohol Villarreal-Vivanco

orden a acotar y zanjar el problema. (Arévalo-Vela, 2010, p. 38). El retardo injustificado en la administración de justicia, imputable en las juezas, jueces, y demás servidoras y servidores de la función judicial y auxiliar de la justicia, será sancionado de conformidad con la ley.

\section{Valoración Crítica de los conceptos principales de las distintas posiciones teóricas sobre el objeto de investigación.}

Entre las opiniones de eminentes conocedores de la carga y celeridad procesal, tenemos:

El tiempo vital, biográfico, existencial es único, de cada uno, de cada yo, y en el que se inserta el proceso que no es impersonal porque lleva dentro de sí a una persona, a esa que la Constitución le garantiza la afectividad de la jurisdicción y la tutela, de una decisión oportuna en tiempo útil. (Morello, 2014, p. 33).

Ha destacado que el Principio de Celeridad Procesal es la consecuencia del concepto que debe tratarse de obtener, el mayor resultado con el mínimo de empleo de actividad procesal, es decir con la debida aplicación del principio de celeridad, se desea lograr que el trabajo del Juez sea menor y el proceso más rápido. "En el proceso el tiempo no es oro, sino Justicia". (García-Véliz, 2016, p. 5)

La celeridad bien puede observarse como uno de los requerimientos primordiales del debido proceso, pues tanto la sociedad como las personas intervinientes en el proceso esperan de la Rama Judicial la definición oportuna de sus peticiones para una convivencia pacífica, confiando en los jueces todas aquellas diferencias surgidas de las interrelaciones familiares, económicas, laborales, (Gallo-Montoya, 2015, p. 12).

Al buscar una mejor administración de justicia lo que se hace es tratar de mejorar todo el sistema judicial y además tratar de que las decisiones que los miembros de la administración de justicia hacen no representen problemas para los individuos una vez que se ha terminado el procedimiento. (Ramírez-Romero, 2015, p. 15). En la actualidad la eficacia de la justicia está ligada a un proceso, que, sin olvidar la aplicación de sus principios como contradicción, igualdad, celeridad y disposición, posibilite una rápida 
lustitia Socialis. Revista Arbitrada de Ciencias Jurídicas.

Año V. Vol. V. N³. Edición Especial. 2020-III:

Universidad Regional Autónoma de los Andes

Hecho el depósito de Ley: FA2016000064

ISSN: 2542-3371

FUNDACIÓN KOINONIA (F.K). Santa Ana de Coro, Venezuela

Iván Xavier León-Rodríguez; Pamela Nicohol Villarreal-Vivanco

solución del conflicto, de modo que la economía y justicia material, conforman los nuevos postulados del Estado Social de Derecho.

Para poder entender de forma más amplia lo que se plantea en esta investigación es necesario remitirnos a los principios que rigen a la función judicial los mismos que se encuentran establecidos en la constitución de la república del ecuador en su Art. 169, en el cual manifiesta que: "Las normas procesales consagran los principios de simplificación, uniformidad, eficacia, inmediación, celeridad y economía procesal..." (Asamblea Nacional Constituyente, 2008, p. 95).

El presente artículo tuvo por objetivo analizar la incidencia en el despacho oportuno de audiencias en materia de Niñez y Adolescencia en contraposición de la carga procesal que tienen los Magistrados del Cantón de Santo Domingo, con el objetivo de determinar si se están cumpliendo los términos establecidos por la ley para la realización de audiencias.

\section{MÉTODO}

Se desarrolló metodológicamente desde una perspectiva descriptiva documental con diseño bibliográfico, lo cual permitió estudiar una población de causas ingresadas en el año 2017 es de 6916, en el año 2018 es de 5818 y en el presente año hasta el mes de julio de 2019 es de 5895 en materia de niñez y adolescencia, procesadas en el Consejo de la Judicatura, en la Unidad Judicial de la Familia, Mujer, Niñez y Adolescencia de Santo Domingo - Ecuador, aplicándose la técnica de análisis jurídico con la finalidad de escrutar las ideas relacionadas con el tema de investigación y así, cumplir con lo propuesto en el objetivo investigativo. 
lustitia Socialis. Revista Arbitrada de Ciencias Jurídicas.

Año V. Vol. V. N³. Edición Especial. 2020-III:

Universidad Regional Autónoma de los Andes

Hecho el depósito de Ley: FA2016000064

ISSN: 2542-3371

FUNDACIÓN KOINONIA (F.K). Santa Ana de Coro, Venezuela

Iván Xavier León-Rodríguez; Pamela Nicohol Villarreal-Vivanco

\section{RESULTADOS}

De acuerdo a los resultados obtenidos por el CJ, tenemos que a inicios del año 2015, la carga que se recibió de años anteriores ascendía a 1'865,381 expedientes carentes de resolución final, y efectuando una proyección a 5 años tendremos una carga procesal atrasada en un 1'000,000 de expedientes pendientes de resolver; significa esto que esta institución se encuentra en una situación de incapacidad para resolver adecuadamente este grave problema, habida cuenta que de los casi 3'000,000 de expedientes en giro, el $30 \%$ se encuentran en ejecución el $70 \%$ se encuentran en trámite; los cuales como ya se explicó líneas arriba se incrementaran en 200,000 al año; notándose claramente que los esfuerzos por incrementar la producción jurisdiccional a través de las políticas instauradas sobre la materia han sido infructuosas para alcanzar una justicia oportuna. Debemos tener en cuenta que el alto número de casos que se manejan en un Juzgado llega a ser asfixiante para el buen funcionamiento del despacho judicial. Esta recarga procesal implica una mayor lentitud en la tramitación de los procesos, lo que significa que debido a esto se proveerán las demandas nuevas así como sus contestaciones en un plazo mucho mayor al estipulado en la norma; se programarán las audiencias en fechas bastante alejadas por motivos de agenda recargada, siendo infructuosos los aislados esfuerzos de algunos jueces de señalar audiencias cada media hora o cada 45 minutos durante todo el día, lo cual no soluciona el problema si no que lo traslada a la falta de tiempo de parte del mismo Juez. 
lustitia Socialis. Revista Arbitrada de Ciencias Jurídicas.

Año V. Vol. V. N³. Edición Especial. 2020-III:

Universidad Regional Autónoma de los Andes

Hecho el depósito de Ley: FA2016000064 ISSN: 2542-3371

FUNDACIÓN KOINONIA (F.K). Santa Ana de Coro, Venezuela

Iván Xavier León-Rodríguez; Pamela Nicohol Villarreal-Vivanco

Tabla 1.

Audiencias realizadas en materia de Familia, Niñez y Adolescencia.

\begin{tabular}{lllll}
\hline Valores & 01 - REALIZADA & 02 - PENDIENTE & 03 - NO REALIZADA & Total Convocadas \\
\hline ene-18 & 746 & 1 & 76 & 823 \\
feb-18 & 658 & - & 60 & 718 \\
mar-18 & 821 & - & 41 & 862 \\
abr-18 & 622 & 1 & 45 & 668 \\
may-18 & 843 & 1 & 119 & 963 \\
jun-18 & 606 & 1 & 55 & 662 \\
jul-18 & 652 & - & 48 & 700 \\
ago-18 & 729 & - & 44 & 773 \\
sep-18 & 708 & - & 93 & 801 \\
oct-18 & 566 & 2 & 105 & 673 \\
nov-18 & 462 & - & 71 & 533 \\
dic-18 & 657 & 1 & 75 & 733 \\
ene-19 & 640 & 1 & 54 & 695 \\
feb-19 & 626 & - & 85 & 711 \\
mar-19 & 338 & - & 72 & 410 \\
abr-19 & 677 & - & 64 & 741 \\
may-19 & 664 & 2 & 86 & 752 \\
jun-19 & 598 & 5 & 87 & 690 \\
jul-19 & 534 & 23 & 91 & 648 \\
\hline
\end{tabular}

Fuente: Dirección Nacional de Estadística (Corte Julio 2019).

Elaborado por: Villareal Vivanco Pamela Nicohol.

Tabla 2

Audiencias realizadas en materia de Familia, Niñez y Adolescencia.

\begin{tabular}{cccccc}
\multirow{2}{*}{ AÑO } & \multirow{2}{*}{ INGRESADAS } & RESUELTAS & \multicolumn{3}{c}{ CAUSAS EN TRÁMITE } \\
& & & INTERMEDIO & TRAMITE & TOTAL GRL \\
\hline $\mathbf{2 0 1 7}$ & 3.732 & 4.869 & 3.381 & 3.535 & 6.916 \\
$\mathbf{2 0 1 8}$ & 3.702 & 4.372 & 2.247 & 3.571 & 5.818 \\
$\mathbf{2 0 1 9}$ & 2.380 & 2.275 & 1.983 & 3.912 & 5.895 \\
\hline
\end{tabular}

Fuente: Dirección Nacional de Estadística (Corte Julio 2019).

Elaborado por: Villareal Vivanco Pamela Nicohol. 
lustitia Socialis. Revista Arbitrada de Ciencias Jurídicas.

Año V. Vol. V. №3. Edición Especial. 2020-III:

Universidad Regional Autónoma de los Andes

Hecho el depósito de Ley: FA2016000064 ISSN: 2542-3371

FUNDACIÓN KOINONIA (F.K). Santa Ana de Coro, Venezuela

Iván Xavier León-Rodríguez; Pamela Nicohol Villarreal-Vivanco

Tabla 3.

Impulsos Procesales.

\begin{tabular}{|l|l|}
\hline PROMEDIO DIARIO* & 40 \\
\hline
\end{tabular}

${ }^{*}$ Se consideran impulsos de escritos, oficios y pagaduría

Fuente: Ing. Christopher Rodas, Coordinador UJ FMNA.

Los impulsos procesales que se dan en coordinación en promedio diario de acuerdo al consejo de la judicatura es de 40, por lo cual a la semana resulta siendo un total de 200 impulsos por causas y al mes un promedio mínimo de 800 no menor a este, manifestando que se da por la carga procesal que existe en dicha materia, para lo cual realizar dicho impulso en coordinación se requiere mínimo realizar dos impulsos en requerimientos y consultas.

\section{DISCUSIÓN}

En la entrevista realizada al Dr. Rodolfo Santillán, Juez de familia, mujer, niñez y adolescencia de Santo Domingo, manifestó que en su cargo se encuentra aproximadamente 7 años laborando como Juez de la Unidad antes mencionada, en su despacho a la semana mantiene un promedio de 100 a 120 causas aproximadamente, de acuerdo a los términos establecidos en el COGEP para la realización de audiencias manifiesta es difícil que se cumplan por la carga procesal que tiene la provincia porque se dan diferentes tipos de audiencias, Audiencias de Conciliación, Audiencias Especiales, Audiencias Únicas, Audiencias Preliminares, Audiencias de Juicio, Audiencias de Juzgamiento entre otras, entonces eso hace complicar que se desarrollen dichas audiencias en el término establecido en el COGEP, como sugerencia para la solución de la carga procesal manifestó que es aumentar el número de jueces en la materia de la investigación y aumentar personal como ayudantes judiciales y secretarios para que se 
lustitia Socialis. Revista Arbitrada de Ciencias Jurídicas.

Año V. Vol. V. N³. Edición Especial. 2020-III:

Universidad Regional Autónoma de los Andes

Hecho el depósito de Ley: FA2016000064 ISSN: 2542-3371

FUNDACIÓN KOINONIA (F.K). Santa Ana de Coro, Venezuela

Iván Xavier León-Rodríguez; Pamela Nicohol Villarreal-Vivanco

cumpla el principio de celeridad y así agilitar el despacho de las causas.

De acuerdo a la (Asamblea Nacional del Ecuador, 2009), por medio del Código Orgánico de la Función Judicial, manifiesta en distintos aspectos la importancia del principio de celeridad procesal como forma de ahorro o economía procesal dentro de todo el desarrollo de un proceso. Entendiendo el Principio de Celeridad Procesal no como un derecho individual de la persona, sino como un instrumento de tutela con rango constitucional que garantiza a todas aquellas personas que concurren ante los órganos jurisdiccionales una justicia equitativa expedita, sin dilaciones indebidas, sin formalismos o reposiciones inútiles.

En tal sentido la celeridad procesal determinada legalmente como principio procesal genera común a todo proceso judicial, constituye deber para un Juez como autoridad del proceso impulsarlo, y secretarios, ayudantes judiciales o demás servidores públicos el cumplimiento de sus funciones facultadas cuya finalidad se tenga la plena finalidad del juicio o proceso que se lleve en la Unidad de familia niñez y adolescencia del cantón Santo Domingo, para la realización de la Justicia por orden Constitucional. (GalarzaVillarruel, 2015, p. 10)

La Importancia de este principio de celeridad procesal es justamente evitar que la carga procesal haga un tardío despacho para el desarrollo de una causa o proceso, siendo base primordial hacer asequible la justicia a los particulares que acuden ante la jurisdicción que a la misma se aplique, en forma breve y rápida, haciendo que se invierta y ahorre tiempo, recursos humanos, económicos en el desarrollo que lleva toda la actividad procesal. (Congo-Suarez, 2015, pág. 8).

\section{CONCLUSIONES}


lustitia Socialis. Revista Arbitrada de Ciencias Jurídicas.

Año V. Vol. V. N³. Edición Especial. 2020-III:

Universidad Regional Autónoma de los Andes

Hecho el depósito de Ley: FA2016000064 ISSN: 2542-3371

FUNDACIÓN KOINONIA (F.K). Santa Ana de Coro, Venezuela

Iván Xavier León-Rodríguez; Pamela Nicohol Villarreal-Vivanco

Se ha evidenciado el incumplimiento de los principios de celeridad, eficacia y eficiencia en la administración de justicia de niñez, esto debido a la excesiva carga procesal asignada a los jueces, que en nuestra provincia la referida unida judicial apenas cuenta con nueve jueces, para atender 4.869 causas anuales.

Se pudo evidenciar que es imperiosa la necesidad del aumento de partidas presupuestarias para contratación de servidores judiciales, con el fin de liberar la carga laboral y conseguir un despacho oportuno de las causas, ofreciéndole al usuario un servicio público eficiente y eficaz.

Es necesario la evaluación constante del desempeño laboral de los servidores judiciales, con el objetivo de determinar el cumplimiento de sus labores de manera oportuna y ágil, aportando de esta manera al desarrollo de una justicia plena y expedita.

\section{FINANCIAMIENTO}

No monetario.

\section{AGRADECIMIENTO}

A la Universidad Regional Autónoma de Los Andes; por motivar el desarrollo de la Investigación.

\section{REFERENCIAS CONSULTADAS}

Arévalo-Vela, J. (2010). El derecho Procesal. Instituto Pacífico.S.A.C.

Asamblea Nacional Constituyente. (2008). Constitución de la República del Ecuador. Montecristi- Ecuador: Corporacion de Estudios y Publicaciones.

Asamblea Nacional del Ecuador. (2009). Código Orgánico de la Función Judicial. QuitoEcuador: Corporacion de Estudios y Publicaciones.

Congo-Suarez, M. (2015). Los principios de celeridad y economía procesal con referencia 
lustitia Socialis. Revista Arbitrada de Ciencias Jurídicas.

Año V. Vol. V. N³. Edición Especial. 2020-III:

Universidad Regional Autónoma de los Andes

Hecho el depósito de Ley: FA2016000064 ISSN: 2542-3371

FUNDACIÓN KOINONIA (F.K). Santa Ana de Coro, Venezuela

Iván Xavier León-Rodríguez; Pamela Nicohol Villarreal-Vivanco

al allanamiento a la demanda Recuperado de http://dspace.utpl.edu.ec/handle/20.500.11962/21733

Galarza-Villarruel, E. (2015). El principio de celeridad y sus efectos jurídicos como garantía básica del debido proceso. Recuperado de http://dspace.uniandes.edu.ec/handle/123456789/2173

Gallo-Montoya, L. A. (2015). Propuestas para Agilizar el Procedimiento. Colombia: Alfaomega.

García-Véliz, L. M. (2016). La Carga Procesal y su Incidencia. Recuperado de https://n9.cl/pisoh

Gutiérrez-Campoverde, H, Cantos-Ludeña, R, \& Durán-Ocampo, A. (2019). Vulneración del debido proceso en el procedimiento penal abreviado [Vulneration of the due process in the abbreviated criminal procedure]. Revista Universidad $y$ Sociedad, 11(4), 414-423.

Hernández-Villareal, G., \& Ortiz, M. (2010). Actualidad y Futuro del Derecho Procesal. Bogotá - Colombia: Universidad del Rosario. Colección textos de jurisprudencia. Primera edición: Bogotá, D.C.

Morello, A. (2014). Doctrina y Jurispudencia Procesal Civil. Argentina: Librería Juris.

Puppio, V. (2012). Teoría General del Proceso. Caracas - Venezuela: Universidad Católica Andrés Bello.

Ramírez-Romero, C. (2015). Principales cuestiones acerca del Código Orgánico General de Procesos. Quito: Corporación de Estudios y Publicaciones. 